OPEN ACCESS

Edited by:

Maria Manuela Rosado, Sapienza University of Rome, Italy

Reviewed by: Marc Hilhorst, Academic Medical Center, Netherlands Jonathan Dick

King's College Hospital NHS Foundation Trust, United Kingdom

*Correspondence: Björn Tampe bjoern.tampe@med.uni-goettingen.de

Specialty section:

This article was submitted to Autoimmune and Autoinflammatory

Disorders,

a section of the journal

Frontiers in Immunology

Received: 20 August 2021 Accepted: 09 September 2021 Published: 30 September 2021

Citation:

Hakroush S and Tampe B (2021) Case Report: ANCA-Associated Vasculitis Presenting With Rhabdomyolysis and Pauci-Immune Crescentic Glomerulonephritis After Pfizer-BioNTech COVID-19 mRNA Vaccination.

Front. Immunol. 12:762006. doi: 10.3389/fimmu.2021.762006

\section{Case Report: ANCA-Associated Vasculitis Presenting With Rhabdomyolysis and Pauci-Immune Crescentic Glomerulonephritis After Pfizer-BioNTech COVID-19 mRNA Vaccination}

\author{
Samy Hakroush ${ }^{1}$ and Björn Tampe ${ }^{2 *}$ \\ ${ }^{1}$ Institute of Pathology, University Medical Center Göttingen, Göttingen, Germany, ${ }^{2}$ Department of Nephrology and \\ Rheumatology, University Medical Center Göttingen, Göttingen, Germany
}

As the coronavirus disease 2019 (COVID-19) pandemic is ongoing and new variants of severe acute respiratory syndrome coronavirus type 2 (SARS-CoV-2) are emerging, there is an urgent need for COVID-19 vaccines to control disease outbreaks by herd immunity. Surveillance of rare safety issues related to these vaccines is progressing, since more granular data emerge with regard to adverse events of COVID-19 vaccines during postmarketing surveillance. Interestingly, four cases of anti-neutrophil cytoplasmic antibody (ANCA)-associated vasculitis (AAV) presenting with pauci-immune crescentic glomerulonephritis (GN) after COVID-19 mRNA vaccination have already been reported. We here expand our current knowledge of this rare but important association and report a case of AAV presenting with massive rhabdomyolysis and pauci-immune crescentic GN after Pfizer-BioNTech COVID-19 mRNA vaccination. As huge vaccination programs are ongoing worldwide, post-marketing surveillance systems must continue to assess vaccine safety important for the detection of any events associated with COVID-19 vaccination. This is especially relevant in complex diseases where diagnosis is often challenging, as in our patient with AAV presenting with massive rhabdomyolysis and pauci-immune crescentic GN.

\footnotetext{
Keywords: coronavirus disease 2019 (COVID-19), vaccination, anti-neutrophil cytoplasmic antibody (ANCA), ANCAassociated vasculitis (AAV), rhabdomyolysis, acute kidney injury (AKI), pauci-immune crescentic glomerulonephritis (GN)
} 


\section{INTRODUCTION}

As the coronavirus disease 2019 (COVID-19) pandemic is ongoing and new variants of severe acute respiratory syndrome coronavirus type 2 (SARS-CoV-2) are emerging, there is an urgent need for COVID-19 vaccines to control disease outbreaks by herd immunity (1). The use of novel vaccines containing a nucleoside-modified messenger ribonucleic acid (mRNA) or a viral deoxyribonucleic acid (DNA) vector that encodes the viral spike (S) glycoprotein of SARS-CoV-2 has already been approved. Large clinical trials have shown that these COVID19 vaccines are safe and effective. Common adverse events include mild to moderate reactions at the injection site, fever, fatigue, body aches, and headache (2). Surveillance of rare safety issues related to these vaccines is progressing, since more granular data emerge with regard to adverse events of COVID19 vaccines during post-marketing surveillance (3). Antineutrophil cytoplasmic antibody (ANCA)-associated vasculitis (AAV) is a small vessel vasculitis hallmarked by the presence of antibodies against autoantigens in cytoplasmic granules of neutrophils (4). AAV presents as granulomatosis with polyangiitis (GPA), microscopic polyangiitis (MPA), and eosinophilic granulomatosis with polyangiitis (EGPA) (5). Generally, renal manifestations in AAV are estimated at $80 \%$ among all cases mainly manifesting as ANCA-associated glomerulonephritis (ANCA GN), and the overall prevalence does not seem to differ substantially between MPO-ANCA and PR3-ANCA AAV (6). Interestingly, five cases of renal AAV presenting with pauci-immune crescentic ANCA GN after COVID-19 mRNA vaccination have already been reported (710). We here expand our current knowledge of this rare but important association and report a case of AAV presenting with massive rhabdomyolysis and pauci-immune crescentic GN after Pfizer-BioNTech COVID-19 mRNA vaccination.

\section{CASE REPORT}

A 79-year-old Caucasian female with a past medical history of hypertension, degenerative disc disease, and no documented history of COVID-19 received two doses of Pfizer-BioNTech COVID-19 mRNA vaccination. Two weeks thereafter, the patient presented to our emergency department with weakness and upper thigh pain. Vital parameters were stable, and physical examination was unremarkable. The patient had no allergies and denied illicit drug use. External routine laboratory assessments obtained 1 week prior to admission were normal for serum creatinine of $0.71 \mathrm{mg} / \mathrm{dl}$ (reference range: $0.5-0.95$ ), estimated glomerular filtration rate (eGFR) of $84.4 \mathrm{ml} / \mathrm{min} / 1.73 \mathrm{~m}^{2}$, and urinalysis with the absence of hematuria or proteinuria. Repeat reverse transcription polymerase chain reaction (RT-PCR) testing for SARS-CoV-2 RNA from nasopharyngeal swabs was negative. Laboratory assessments at admission showed massive rhabdomyolysis with creatinine kinase (CK) levels of 14,243 U/L (reference range: 29-168), myoglobinemia of $>12,000 \mu \mathrm{g} / \mathrm{L}$ (reference range: $\leq 106$, Figure 1A), and acute kidney injury (AKI) with serum creatinine levels of $1.38 \mathrm{mg} / \mathrm{dl}$ (reference range: $0.7-1.2$, Figure 1B) and an estimated glomerular filtration rate (eGFR) of $33.5 \mathrm{ml} / \mathrm{min} / 1.73 \mathrm{~m}^{2}$. Urinary analysis revealed leukocyturia, hematuria (no dysmorphic erythrocytes), few renal tubular epithelial cells, and nephrotic range proteinuria of $>18,000 \mathrm{mg} / \mathrm{g}$ creatinine and albuminuria of $<5,000 \mathrm{mg} / \mathrm{g}$ creatinine (reference range: $<30 \mathrm{mg} / \mathrm{g}$, Figure 1C). The patient received intravenous crystalloids with decreasing CK levels and myoglobinemia (Figure 1A). However, progressive deterioration of kidney function with worsening of serum creatinine levels up to $6.57 \mathrm{mg} / \mathrm{dl}$ (reference range: $0.7-1.2 \mathrm{mg} / \mathrm{dl}$, Figure $1 \mathrm{~B}$ ) and an eGFR of $<15 \mathrm{ml} / \mathrm{min} / 1.73 \mathrm{~m}^{2}$ occurred. ANCA immunofluorescence (IF) was positive at 1:1,000 (reference range: <1:10) with elevated MPO-ANCA levels >134 IU/ml (reference range: $<3.5 \mathrm{IU} / \mathrm{ml}$ ), while myositis antibodies, complement levels, and other serologic parameters were all tested negative (Table 1). Because of leukocytosis, a white blood differential was conducted revealing prominent peripheral blood eosinophilia (Table 1).

Based on suspected MPO-positive AAV, the patient received a steroid pulse with intravenous methylprednisolone for 3 days (250 mg per day) and oral prednisone $1 \mathrm{mg} / \mathrm{kg}$ daily thereafter (60 mg per day, Figure 1D). A kidney biopsy confirmed severe acute tubular injury with pauci-immune crescentic GN and interstital nephritis: cellular crescents in $1 / 15(6.7 \%)$ glomeruli, global glomerular sclerosis in $2 / 15$ (13.3\%), mild (5\%) interstitial fibrosis and tubular atrophy (IF/TA), interstitial inflammation (25\%) with prominent eosinophilic infiltration, and severe acute tubular injury with myoglobin casts (Figure 2 and Table 2). According to histopathological scoring, focal class ANCA GN and intermediate risk ANCA renal risk score (ARRS) were present (Table 2) $(11,12)$. Based on the diagnosis of AAV presenting with massive rhabdomyolysis and pauci-immune crescentic GN, intravenous cyclophosphamide (CYC) was initiated at $10 \mathrm{mg} / \mathrm{kg}$ (per CYCLOPS trial dosing, Figure 1D) (13). Thereafter, kidney function normalized without requirement of dialysis and proteinuria decreased to $1,603 \mathrm{mg} / \mathrm{g}$ creatinine and albuminuria to $351 \mathrm{mg} / \mathrm{g}$ creatinine (reference range: $<30 \mathrm{mg} / \mathrm{g}$, Figures 1B, C). Repeat serological testing confirmed that ANCA IF turned negative. Thereafter, oral prednisone was tapered down (currently $50 \mathrm{mg}$ per day), and we do not plan to repeat administration of intravenous cyclophosphamide because rhabdomyolysis ceased and kidney function normalized.

\section{DISCUSSION}

To our knowledge, we here present the first case of AAV presenting with massive rhabdomyolysis and pauci-immune crescentic GN after Pfizer-BioNTech COVID-19 mRNA vaccination. With millions of people being vaccinated for COVID-19, rare reports of adverse events are emerging. In this case, the temporal association between Pfizer-BioNTech 
Days after admission

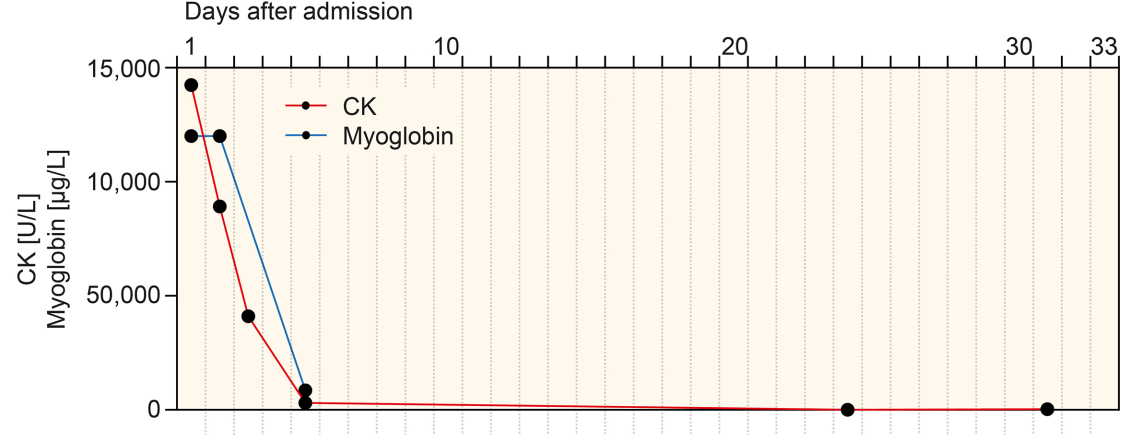

B
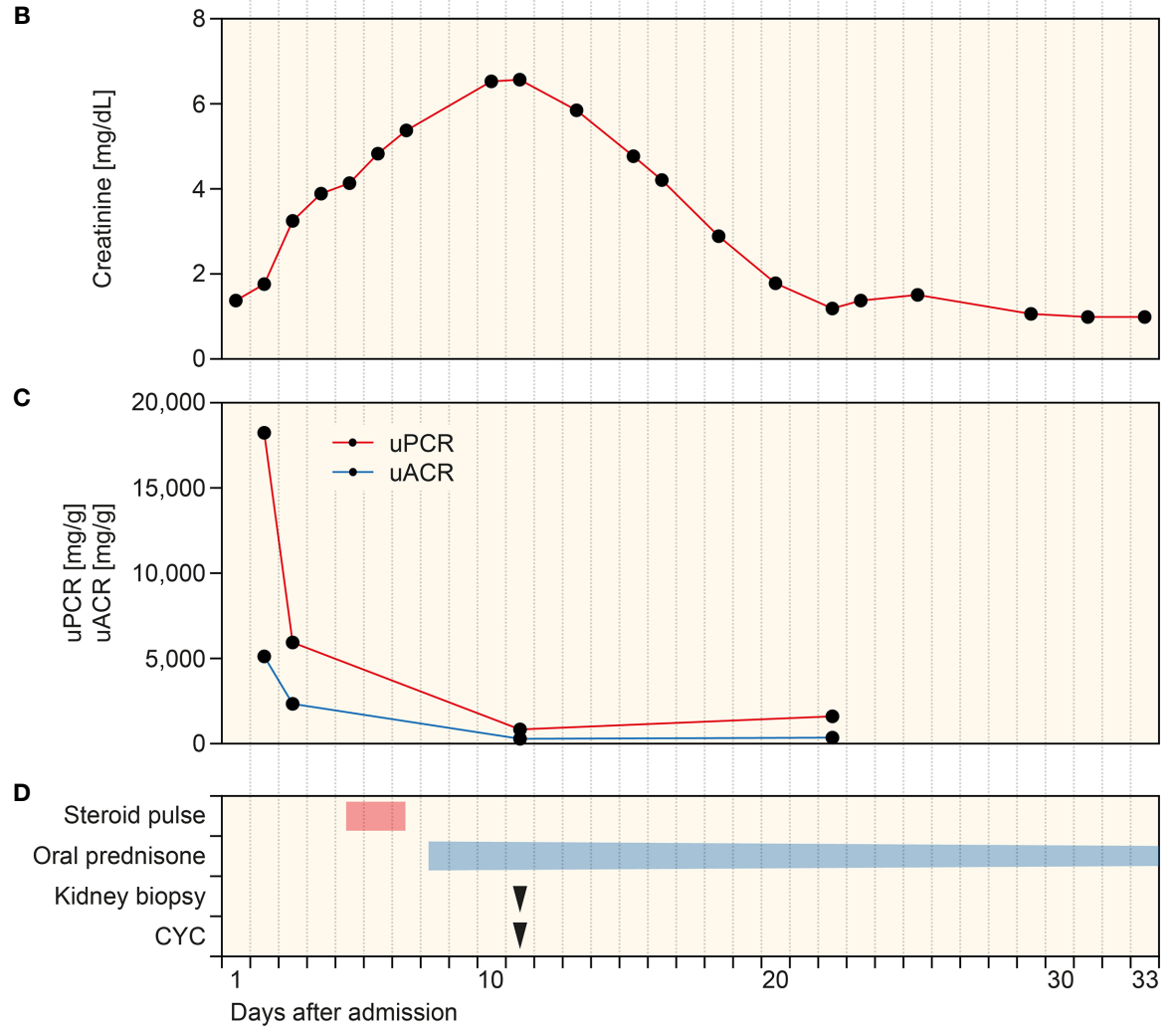

FIGURE 1 | Timeline of the case after admission. (A-C) Time course of CK, myoglobin, plasma creatinine, and levels of uPCR and uACR. (D) Time of treatment regimens and kidney biopsy. CK, creatinine kinase: CYC, cyclophosphamide; UACR, urinary albumin-to-creatinine ratio; UPCR, urinary protein-to-creatinine ratio. 
TABLE 1 | Serologic parameters after admission.

\begin{tabular}{|c|c|c|}
\hline & Value & $\begin{array}{c}\text { Reference } \\
\text { range }\end{array}$ \\
\hline \multicolumn{3}{|l|}{ Serologic parameters } \\
\hline HIV Ag/Ab-titer & Neg & Neg \\
\hline HBsAg-titer & Neg & Neg \\
\hline Anti-HCV-titer & Neg & Neg \\
\hline Rheumatoid factor - IU/ml & $<10$ & $<15.9$ \\
\hline Complement C3c-g/L & 0.97 & $0.82-1.93$ \\
\hline Complement C4-g/L & 0.19 & $0.15-0.57$ \\
\hline ANCA IF & $1: 1,000$ & $<1: 10$ \\
\hline PR3-ANCA-IU/ml & $<0.2$ & $<2$ \\
\hline MPO-ANCA-IU/ml & $>134$ & $<3.5$ \\
\hline ENA screen & $<0.1$ & $<0.7$ \\
\hline Anti-DFS70-U/ml & $<0.6$ & $<7$ \\
\hline Anti-ds-DNA-IU/ml & 4.4 & $<15$ \\
\hline Histones - U/ml & 7.7 & $<20$ \\
\hline ANA IF & $1: 320$ & $<1: 100$ \\
\hline R052-blot & Neg & Neg \\
\hline PM-Scl-100-blot & Neg & Neg \\
\hline PM-Scl-75-blot & Neg & Neg \\
\hline $\mathrm{Ku}-\mathrm{blot}$ & Neg & Neg \\
\hline SRP-blot & Neg & Neg \\
\hline PL-7-blot & $\mathrm{Neg}$ & Neg \\
\hline PL-12-blot & Neg & Neg \\
\hline EJ-blot & Neg & Neg \\
\hline OJ-blot & Neg & Neg \\
\hline JO1-blot & Neg & Neg \\
\hline Mi alpha-blot & Neg & Neg \\
\hline Mi-2 beta-blot & Neg & Neg \\
\hline TIF1 gamma-blot & Neg & Neg \\
\hline MDA-5-blot & Neg & Neg \\
\hline NXP2-blot & Neg & Neg \\
\hline SAE1-blot & Neg & Neg \\
\hline \multicolumn{3}{|l|}{ White blood differential } \\
\hline 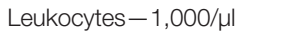 & 22.9 & $4-11$ \\
\hline Lymphocytes-\% & 4.7 & $20-45$ \\
\hline Monocytes-\% & 4.5 & $3-13$ \\
\hline Eosinophils-\% & 23.3 & $\leq 8$ \\
\hline Basophils-\% & 0.2 & $\leq 2$ \\
\hline Neutrophils-\% & 67.3 & $40-76$ \\
\hline
\end{tabular}

ANA, antinuclear antibodies; ANCA, anti-neutrophil cytoplasmic antibody; ds-DNA, double stranded-DNA; DSF70, dense-fine-speckled 70; ENA, EJ, glycine; ENA, extractable nuclear antigen; HBsAg, hepatitis B surface antigen; HCV, hepatitis C virus; HIV, human immunodeficiency virus; JO1, histidyl tRNA synthetase; MDA-5, melanoma differentiation-associated protein-5; MPO, myeloperoxidase; Neg, negative; NXP2, nuclear matrix protein 2; OJ, isoleucine; PM-SCl, PL-7, threonine; PL-12, alanine, polymyositis-scleroderma; PR3, proteinase 3; SAE1, small ubiquitin-like modifier activating enzyme; SRP, signal recognition particle; TIF1, transcriptional intermediary factor 1 .

COVID-19 mRNA vaccination and AAV presenting with rhabdomyolysis and pauci-immune crescentic GN suggests a neutrophilic immune response to mRNA as a potential trigger. This patient initially presented with upper thigh pain due to massive rhabdomyolysis after Pfizer-BioNTech COVID-19 mRNA vaccination. Rhabdomyolysis has been described in the context of COVID-19, and a direct viral tropism to myocytes has been suggested $(14,15)$. However, detection of SARS-CoV-2 infection in skeletal muscle cells has not been established yet (16). Rhabdomyolysis secondary to vaccination has previously been reported, mostly in the context of influenza vaccination (17, 18). In association with COVID-19 mRNA vaccination, the onset of fatigue, myalgias, and arthralgias following mRNA vaccination has been reported in a considerable subset of patients (19). Additionally, there is evidence that COVID-19 mRNA vaccination can directly induce myositis at the injection site, as previously observed in the deltoid muscle (20). In addition to rhabdomyolysis, we observed pauci-immune crescentic GN accompanied by MPO-ANCA autoantibodies after COVID-19 mRNA vaccination. To date, five cases of pauci-immune crescentic ANCA GN after the second dose of COVID-19 mRNA vaccination in all cases have been reported (7-10). In our case, kidney biopsy showed myoglobin casts due to massive rhabdomyolysis and pauci-immune crescentic GN, and it is likely that both contributed to deterioration of kidney function. It has already been reported that the first COVID-19 mRNA vaccination primes the innate immune system to mount a more potent response after the second booster immunization (21). It is possible that the enhanced immune response especially observed after the second dose of COVID-19 mRNA vaccination could be responsible for triggering the observed MPO-ANCA autoantibodies. Causal links between immune system activation by viral infections and AAV have been suggested due to onset of AAV predominantly during the winter (22, 23). Toll-like receptors (TLRs) are expressed on leukocytes and play crucial roles in the recognition of viral antigens, facilitating immune system activation and inflammation. Predominant TLR-2 and TLR-9 activation can stimulate autoimmunity in AAV, previously been described in the context of MPO-ANCA autoantibodies (24). Interestingly, TLR-2 activation in immunodominant cytotoxic $\mathrm{T}$ lymphocytes in response to SARS-CoV-2 S glycoprotein (as also produced by COVID-19 vaccines) has already been described (25). With regard to vaccination, there is some discussion about the relationship between vaccination and AAV recurrence in patients with preexisting autoimmune disease after influenza vaccination as very rare but significant side effects (26). The temporal relationship could be explained theoretically, including molecular mimicry, polyclonal activation, or transient systemic proinflammatory cytokine responses that potentially provoke autoimmune diseases in genetically predisposed individuals (27). Interestingly, increased production of ANCA autoantibodies has already been described in response to viral mRNA-based influenza and rabies vaccines (27). Moreover, AAV and autoimmune reactions have been reported in the context of COVID-19, implicating a direct reaction to viral RNA (28-30). Therefore, the occurrence of AAV in the context of COVID-19 mRNA as compared with non-mRNA vaccines would be of great relevance. Huge vaccination programs are ongoing worldwide, and post-marketing surveillance systems must continue to assess vaccine safety important for the detection of any events associated with COVID-19 vaccination. This is especially relevant in complex diseases where diagnosis is often challenging, as in our patient with AAV presenting with massive rhabdomyolysis and pauci-immune crescentic GN. The limitation of this case report is only a temporal relationship between COVID-19 mRNA vaccination and onset of AAV. However, AAV onset in association with COVID-19 

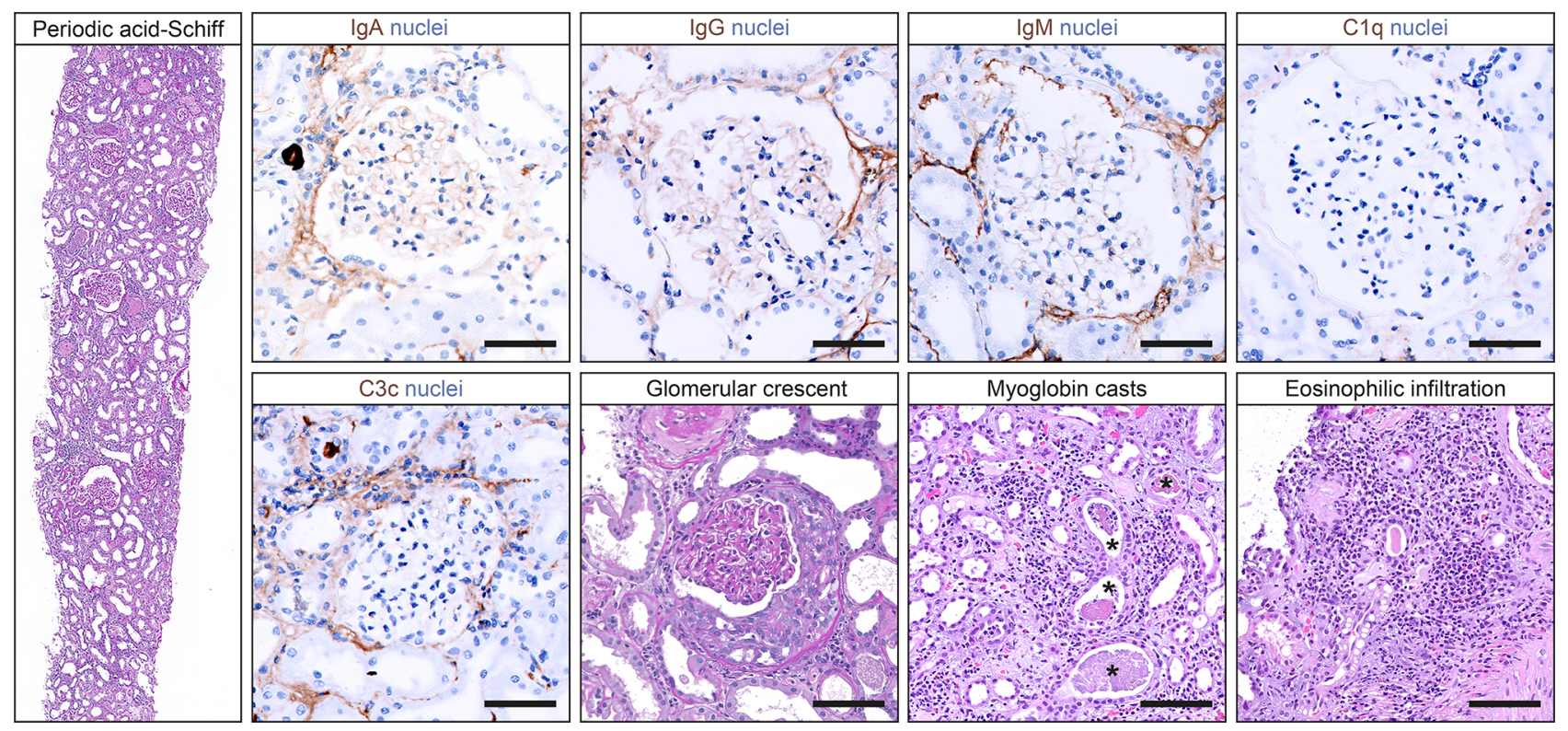

FIGURE 2 | Histopathological findings in a kidney biopsy confirming pauci-immune crescentic GN. Representative photomicrographs of the kidney biopsy including staining for IgA (scale bar: $50 \mu \mathrm{m}$ ), IgG (scale bar: $50 \mu \mathrm{m}$ ), IgM (scale bar: $50 \mu \mathrm{m}$ ), C1q (scale bar: $50 \mu \mathrm{m}$ ), and C3c (scale bar: $50 \mu \mathrm{m}$ ); periodic acid-Schiff staining showing a glomerulus with crescent formation (scale bar: $50 \mu \mathrm{m}$ ); and hematoxylin/eosin staining with myoglobin casts (asterisks, scale bar: $100 \mu \mathrm{m}$ ) and tubulointerstitial inflammation with prominent eosinophilic infiltration (scale bar: $100 \mu \mathrm{m}$ ). C1q, complement component 1q; C3c, complement factor 3 conversion product; IgA, immunoglobulin A; IgG, immunoglobulin G; IgM, immunoglobulin M; GN, glomerulonephritis.

TABLE 2 | Histopathological findings in the kidney biopsy

\begin{tabular}{lc}
\hline & Value \\
\hline Lesions & \\
Total glomeruli-no. & 15 \\
Cellular crescents-no. (\%) & $1(6.7)$ \\
Fibrocellular crescents-no. (\%) & $0(0)$ \\
Global glomerular sclerosis-no. (\%) & $2(13.3)$ \\
IF/TA-\% & 5 \\
Interstitial inflammation-\% & 25 \\
Scoring & \\
Berden class & Focal \\
ARRS & Intermediate risk \\
\hline ARRS, ANCA renal risk score: IF/TA, interstitial fibrosis/tubular atrophy; no., number.
\end{tabular}

mRNA vaccination has independently been observed before and requires further investigation with regard to the mechanisms linking autoimmunity to COVID-19 vaccines (7-9). Fortunately, treatment of AAV is possible and caution in such cases is warranted with regard to early testing if clinical symptoms are compatible with AAV in principle.

\section{DATA AVAILABILITY STATEMENT}

The original contributions presented in the study are included in the article/supplementary material. Further inquiries can be directed to the corresponding author.

\section{ETHICS STATEMENT}

Ethical review and approval was not required for the study on human participants in accordance with the local legislation and institutional requirements. The patients/participants provided their written informed consent to participate in this study.

\section{AUTHOR CONTRIBUTIONS}

BT was directly involved in the treatment of the patient, conceived the case report, collected and analyzed the data, and wrote the manuscript. SH evaluated kidney biopsy findings and edited the manuscript. All authors contributed to the article and approved the submitted version.

\section{FUNDING}

We acknowledge the support from the Open Access Publication Funds of the Georg August University Göttingen.

\section{ACKNOWLEDGMENTS}

The authors thank the treating medical staff involved in treating the patient at our hospital. 


\section{REFERENCES}

1. Garcia-Montero C, Fraile-Martinez O, Bravo C, Torres-Carranza D, SanchezTrujillo L, Gómez-Lahoz AM, et al. An Updated Review of SARS-CoV-2 Vaccines and the Importance of Effective Vaccination Programs in Pandemic Times. Vaccines (Basel) (2021) 9(5):433. doi: 10.3390/vaccines 9050433

2. Polack FP, Thomas SJ, Kitchin N, Absalon J, Gurtman A, Lockhart S, et al. Safety and Efficacy of the BNT162b2 mRNA Covid-19 Vaccine. N Engl J Med (2020) 383(27):2603-15. doi: 10.1056/NEJMoa2034577

3. Pollard AJ, Bijker EM. A Guide to Vaccinology: From Basic Principles to New Developments. Nat Rev Immunol (2021) 21(2):83-100. doi: 10.1038/s41577020-00479-7

4. Jennette JC, Nachman PH. ANCA Glomerulonephritis and Vasculitis. Clin J Am Soc Nephrol (2017) 12(10):1680-91. doi: 10.2215/CJN.02500317

5. Falk RJ, Jennette JC. Anti-Neutrophil Cytoplasmic Autoantibodies With Specificity for Myeloperoxidase in Patients With Systemic Vasculitis and Idiopathic Necrotizing and Crescentic Glomerulonephritis. $N$ Engl J Med (1988) 318(25):1651-7. doi: 10.1056/NEJM198806233182504

6. Franssen C, Gans R, Kallenberg C, Hageluken C, Hoorntje S. Disease Spectrum of Patients With Antineutrophil Cytoplasmic Autoantibodies of Defined Specificity: Distinct Differences Between Patients With AntiProteinase 3 and Anti-Myeloperoxidase Autoantibodies. J Intern Med (1998) 244(3):209-16. doi: 10.1046/j.1365-2796.1998.00357.x

7. Sekar A, Campbell R, Tabbara J, Rastogi P. ANCA Glomerulonephritis After the Moderna COVID-19 Vaccination. Kidney Int (2021) 100(2):473-4. doi: 10.1016/j.kint.2021.05.017

8. Anderegg MA, Liu M, Saganas C, Montani M, Vogt B, Huynh-Do U, et al. De Novo Vasculitis After mRNA-1273 (Moderna) Vaccination. Kidney Int (2021) 100(2):474-6. doi: 10.1016/j.kint.2021.05.016

9. Shakoor MT, Birkenbach MP, Lynch M. ANCA-Associated Vasculitis Following Pfizer-BioNTech COVID-19 Vaccine. Am J Kidney Dis (2021) 78 (4):611-3. doi: 10.1053/j.ajkd.2021.06.016

10. Dube GK, Benvenuto LJ, Batal I. ANCA-Associated Glomerulonephritis Following the Pfizer-BioNTech COVID-19 Vaccine. Kidney Int Rep (2021). doi: 10.1016/j.ekir.2021.08.012

11. Berden AE, Ferrario F, Hagen EC, Jayne DR, Jennette JC, Joh K, et al. Histopathologic Classification of ANCA-Associated Glomerulonephritis. J Am Soc Nephrol (2010) 21(10):1628-36. doi: 10.1681/ASN.2010050477

12. Brix SR, Noriega M, Tennstedt P, Vettorazzi E, Busch M, Nitschke M, et al. Development and Validation of a Renal Risk Score in ANCA-Associated Glomerulonephritis. Kidney Int (2018) 94(6):1177-88. doi: 10.1016/ j.kint.2018.07.020

13. de Groot K, Harper L, Jayne DRW, Suarez LFF, Gregorini G, Gross WL, et al. Pulse Versus Daily Oral Cyclophosphamide for Induction of Remission in Antineutrophil Cytoplasmic Antibody-Associated Vasculitis: A Randomized Trial. Ann Intern Med (2009) 150(10):670-80. doi: 10.7326/0003-4819-15010-200905190-00004

14. Mack M, Nichols L, Guerrero DM. Rhabdomyolysis Secondary to COVID-19 Vaccination. Cureus (2021) 13(5):e15004. doi: 10.7759/cureus.15004

15. Haroun MW, Dieiev V, Kang J, Barbi M, Nia SFM, Gabr M, et al. Rhabdomyolysis in COVID-19 Patients: A Retrospective Observational Study. Cureus (2021) 13(1):e12552. doi: 10.7759/cureus.12552

16. Ding Y, He L, Zhang Q, Huang Z, Che X, Hou J, et al. Organ Distribution of Severe Acute Respiratory Syndrome (SARS) Associated Coronavirus (SARS-CoV) in SARS Patients: Implications for Pathogenesis and Virus Transmission Pathways. J Pathol (2004) 203(2):622-30. doi: 10.1002/path.1560

17. Callado RB, Carneiro TG, Parahyba CC, Lima Nde A, da Silva Junior GB, Daher Ede F. Rhabdomyolysis Secondary to Influenza A H1N1 Vaccine Resulting in Acute Kidney Injury. Travel Med Infect Dis (2013) 11(2):130-3. doi: 10.1016/j.tmaid.2012.11.004

18. Rajaratnam N, Govil S, Patel R, Ahmed M, Elias S. Rhabdomyolysis After Recombinant Zoster Vaccination: A Rare Adverse Reaction. J Community
Hosp Intern Med Perspect (2021) 11(1):145-6. doi: 10.1080/20009666. 2020.1841878

19. Baden LR, El Sahly HM, Essink B, Kotloff K, Frey S, Novak R, et al. Efficacy and Safety of the mRNA-1273 SARS-CoV-2 Vaccine. N Engl J Med (2021) 384 (5):403-16. doi: 10.1056/NEJMoa2035389

20. Theodorou DJ, Theodorou SJ, Axiotis A, Gianniki M, Tsifetaki N. COVID-19 Vaccine-Related Myositis. QJM (2021) hcab043. doi: 10.1093/qjmed/hcab043

21. Arunachalam PS, Scott MKD, Hagan T, Li C, Feng Y, Wimmers F, et al. Systems Vaccinology of the BNT162b2 mRNA Vaccine in Humans. Nature (2021) 596(7872):410-6. doi: 10.1038/s41586-021-03791-x

22. Konstantinov KN, Emil SN, Barry M, Kellie S, Tzamaloukas AH. Glomerular Disease in Patients With Infectious Processes Developing Antineutrophil Cytoplasmic Antibodies. ISRN Nephrol (2013) 2013:324315. doi: 10.5402/ 2013/324315

23. Tidman M, Olander R, Svalander C, Danielsson D. Patients Hospitalized Because of Small Vessel Vasculitides With Renal Involvement in the Period 1975-95: Organ Involvement, Anti-Neutrophil Cytoplasmic Antibodies Patterns, Seasonal Attack Rates and Fluctuation of Annual Frequencies. J Intern Med (1998) 244(2):133-41. doi: 10.1046/j.1365-2796.1998.00324.x

24. Summers SA, Steinmetz OM, Gan PY, Ooi JD, Odobasic D, Kitching AR, et al. Toll-Like Receptor 2 Induces Th17 Myeloperoxidase Autoimmunity While Toll-Like Receptor 9 Drives Th1 Autoimmunity in Murine Vasculitis. Arthritis Rheumatol (2011) 63(4):1124-35. doi: 10.1002/art.30208

25. Kumar N, Admane N, Kumari A, Sood D, Grover S, Prajapati VK, et al. Cytotoxic T-Lymphocyte Elicited Vaccine Against SARS-CoV-2 Employing Immunoinformatics Framework. Sci Rep (2021) 11(1):7653. doi: 10.1038/ s41598-021-86986-6

26. Jeffs LS, Peh CA, Jose MD, Lange K, Hurtado PR. Randomized Trial Investigating the Safety and Efficacy of Influenza Vaccination in Patients With Antineutrophil Cytoplasmic Antibody-Associated Vasculitis. Nephrol (Carlton) (2015) 20(5):343-51. doi: 10.1111/nep.12416

27. Jeffs LS, Nitschke J, Tervaert JW, Peh CA, Hurtado PR. Viral RNA in the Influenza Vaccine may Have Contributed to the Development of ANCAAssociated Vasculitis in a Patient Following Immunisation. Clin Rheumatol (2016) 35(4):943-51. doi: 10.1007/s10067-015-3073-0

28. Uppal NN, Kello N, Shah HH, Khanin Y, De Oleo IR, Epstein E, et al. De Novo ANCA-Associated Vasculitis With Glomerulonephritis in COVID-19. Kidney Int Rep (2020) 5(11):2079-83. doi: 10.1016/j.ekir.2020.08.012

29. Izci Duran T, Turkmen E, Dilek M, Sayarlioglu H, Arik N. ANCA-Associated Vasculitis After COVID-19. Rheumatol Int (2021) 41(8):1523-9. doi: 10.1007/ s00296-021-04914-3

30. Vlachoyiannopoulos PG, Magira E, Alexopoulos H, Jahaj E, Theophilopoulou $\mathrm{K}$, Kotanidou A, et al. Autoantibodies Related to Systemic Autoimmune Rheumatic Diseases in Severely Ill Patients With COVID-19. Ann Rheum Dis (2020) 79(12):1661-3. doi: 10.1136/annrheumdis-2020-218009

Conflict of Interest: The authors declare that the research was conducted in the absence of any commercial or financial relationships that could be construed as a potential conflict of interest.

Publisher's Note: All claims expressed in this article are solely those of the authors and do not necessarily represent those of their affiliated organizations, or those of the publisher, the editors and the reviewers. Any product that may be evaluated in this article, or claim that may be made by its manufacturer, is not guaranteed or endorsed by the publisher.

Copyright (c) 2021 Hakroush and Tampe. This is an open-access article distributed under the terms of the Creative Commons Attribution License (CC BY). The use, distribution or reproduction in other forums is permitted, provided the original author(s) and the copyright owner(s) are credited and that the original publication in this journal is cited, in accordance with accepted academic practice. No use, distribution or reproduction is permitted which does not comply with these terms. 